\title{
TREATMENT OF PERIPHERAL FACIAL NERVE PALSY WITH ACUPUNCTURE, COMPLEMENTARY OR ALTERNATIVE TO CONVENTIONAL PHARMACEUTICAL TREATMENT
}

Sofia Ferfeli ${ }^{1}$, Damiani Tsiamasfirou ${ }^{1}$, Panagiotis Vorniotakis ${ }^{1}$, Sofia Sivetidou ${ }^{1}$, Aikaterini Kotroni1 ${ }^{1}$, Eleutherios Bakas ${ }^{1}$ 'Physical Medicine and Rehabilitation department, KAT Hospital- Athens, Greece

INTRODUCTION: Peripheral facial nerve palsy is a common occurrence in both the emergency room and the neurology outpatient clinical setting. It is usually treated pharmaceutically in the acute phase, with per os methylprednisolone, anti-herpetic agents and topical ocular ointments.

PURPOSE: To determine the efficiency of medical acupuncture on peripheral facial nerve palsy patients, either as a complementary form of therapy or as a primary form in the acute phase, on those with contraindications to receive corticosteroids.

METHOD: A group of 6 patients were selected to receive acupuncture treatment twice a week, for a total of 12 sessions. Five of those patients had received a full course of per os and topical treatment 2 to 13 months earlier. One of the patients had a history of diabetes mellitus type I and hepatitis and was contraindicated from receiving steroid treatment, therefore he underwent acupuncture sessions in the acute phase, along with topical eye treatment.

All patients were given instructions to follow a six week home based mime therapy program, which consisted of automassage, coordination and emotional expression exercises. The efficacy of the treatment was recorded using the Facial Disability Index (FDI) and for those who experienced pain, the Numeric Pain Rating Scale (NPRS), before and after the conclusion of the six week protocol.

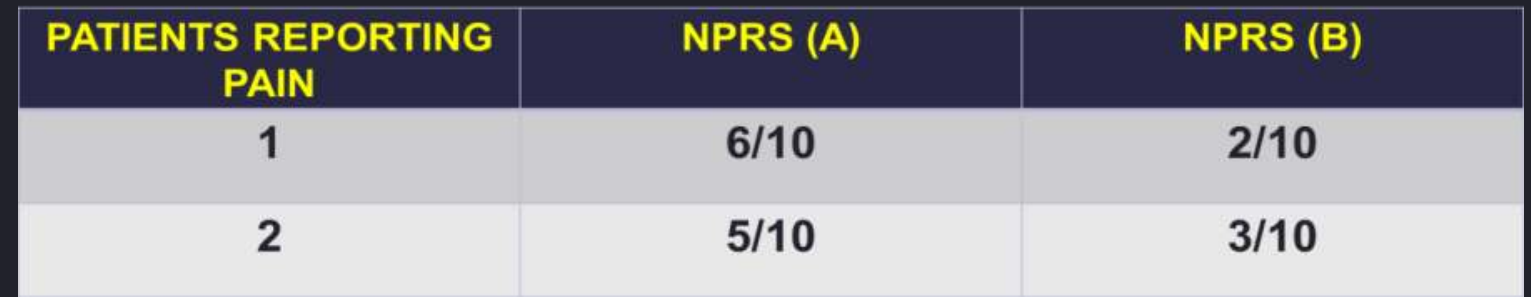

Numeric Pain Rating Scale scores before (A) and after (B) the six week acupunture protocoll.
RESULTS: Concluding the six week acupuncture treatment all six patients reported improvement in both physical and social function on the Facial Disability Index. The two subscales of the FDI are being recorded separately, since according to the literature, are not necessarily associated. Those patients who experienced pain noted pain reduction on the NPRS.

CONCLUSIONS: Acupuncture is a form of therapy for peripheral facial nerve palsy which seems to be effective in our patients, whether as an adjuvant to pharmaceutical therapy or as a primary therapy, always in the setting of a multidimensional rehabilitation program.

\begin{tabular}{l|l|l|l} 
PATIENTS & PHYSICAL & PHYSICAL & SOCIAL/ WELL SOCIAL/ WELL
\end{tabular} FUNCTION (A) FUNCTION (B) BEING BEING FUNCTION (A) FUNCTION (B)

\begin{tabular}{|l|l|l|l|l|}
\hline 1 & 50 & 95 & 76 & 92 \\
\hline 2 & 75 & 85 & 12 & 16 \\
\hline 3 & 40 & 75 & 28 & 32 \\
\hline 4 & 60 & 80 & 80 & 88 \\
\hline 5 & 40 & 55 & 56 & 60 \\
\hline 6 & 85 & 95 & 92 & 96 \\
\hline
\end{tabular}

The Facial Disability Index (FDI) with two subscales: Physical Function Kal Social/ Well Being Function before (A) and after (B) the six week therapeutic intervention. The scores represent percent functional ability.

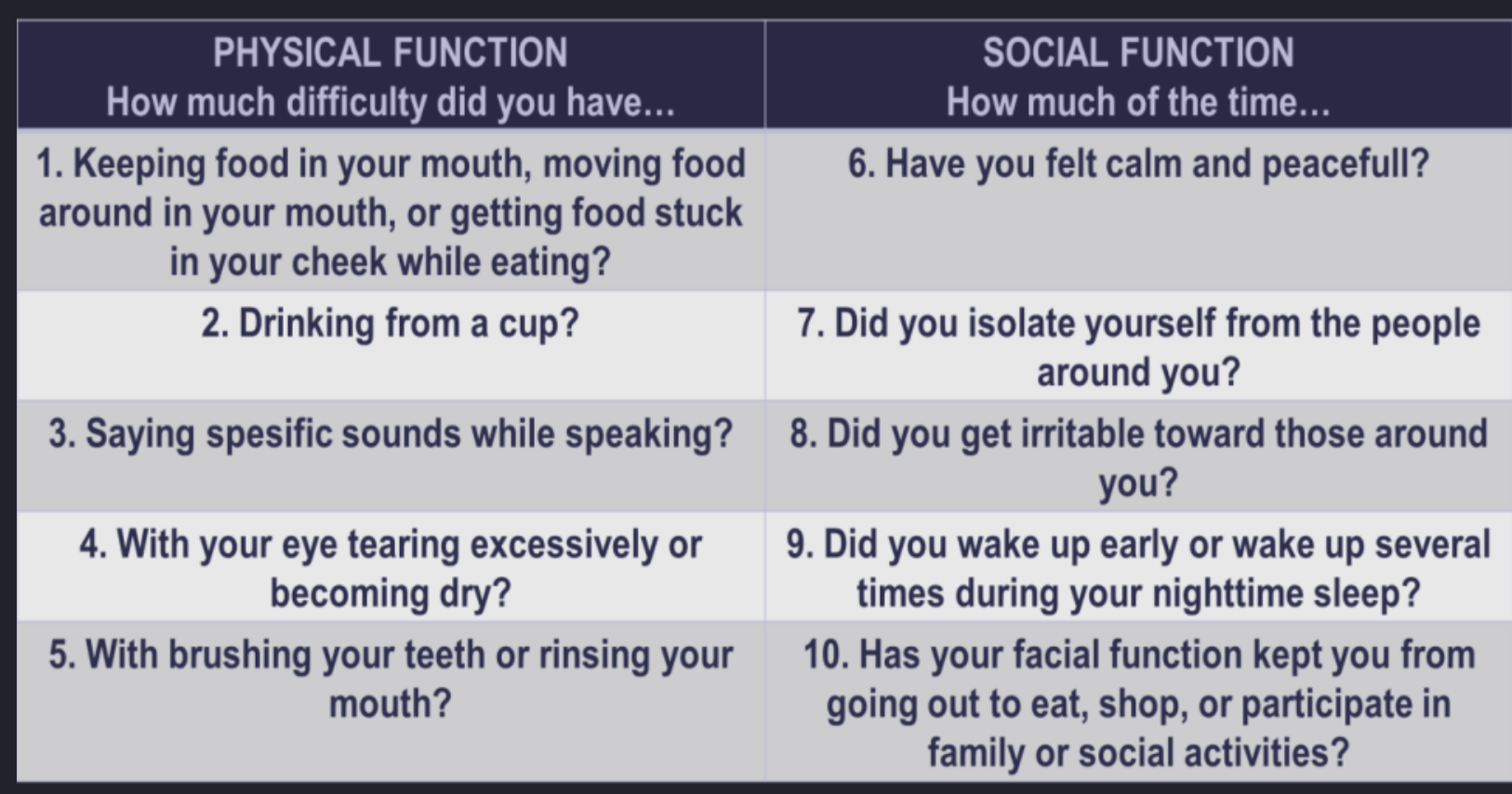

The Facial Disability Index 